\title{
A new smoothness result for Caputo-type fractional ordinary differential equations *
}

\author{
Binjie Li † Xiaoping Xie $\ddagger$ Shiquan Zhang $§$ \\ School of Mathematics, Sichuan University, Chengdu 610064, China
}

\begin{abstract}
We present a new smoothness result for Caputo-type fractional ordinary differential equations, which reveals that, subtracting a non-smooth function that can be obtained by the information available, a non-smooth solution belongs to $C^{m}$ for some positive integer $m$.
\end{abstract}

Keywords: Caputo, fractional differential equation, smoothness.

\section{Introduction}

Let us consider the following model problem: seek $0<h \leqslant a$ and

$$
y \in\left\{v \in C[0, h]:\left\|v-c_{0}\right\|_{C[0, h]} \leqslant b\right\}
$$

such that

$$
\left\{\begin{array}{l}
D_{*}^{\alpha} y=f(x, y), \quad 0 \leqslant x \leqslant h, \\
y(0)=c_{0},
\end{array}\right.
$$

where $a>0, b>0,0<\alpha<1, c_{0} \in \mathbb{R}$, and

$$
f \in C\left([0, a] \times\left[c_{0}-b, c_{0}+b\right]\right) .
$$

Above, the Caputo-type fractional differential operator $D_{*}^{\alpha}: C[0, h] \rightarrow C_{0}^{\infty}(0, h)^{\prime}$ is given by

$$
D_{*}^{\alpha} z:=D J^{1-\alpha}(z-z(0))
$$

for all $z \in C[0, h]$, where $D$ denotes the well-known first order generalized differential operator, and the Riemann-Liouville fractional integral operator $J^{1-\alpha}: C[0, h] \rightarrow C[0, h]$ is defined by

$$
J^{1-\alpha} z(x):=\frac{1}{\Gamma(1-\alpha)} \int_{0}^{x}(x-t)^{-\alpha} z(t) \mathrm{d} t, \quad 0 \leqslant x \leqslant h,
$$

for all $z \in C[0, h]$.

By [2, Lemma 2.1], the above problem is equivalent to seeking solutions of the following Volterra integration equation:

$$
y(x)=c_{0}+\frac{1}{\Gamma(\alpha)} \int_{0}^{x}(x-t)^{\alpha-1} f(t, y(t)) \mathrm{d} t .
$$

Diethelm and Ford [2] proved that, if $f$ is continuous, then (1.3) has a solution $y \in C[0, h]$ for some $0<h \leqslant a$, and this solution is unique if $f$ is Lipschitz continuous. A natural question arises whether $y$ can be smoother than being continuous. This is not only of theoretical value, but also of great importance in developing numerical methods for (1.3).

*This work was supported in part by Major Research Plan of National Natural Science Foundation of China (91430105) and National Natural Science Foundation of China (11401407).

${ }^{\dagger}$ Email: libinjiefem@yahoo.com

${ }^{\ddagger}$ Corresponding author. Email: xpxie@scu.edu.cn

§Email: shiquanzhang@scu.edu.cn 
To this question, Miller and Feldstein [5] gave the first answer: if $f$ is analytic, then $y$ is analytic in $(0, h)$ for some $0<h \leqslant a$. Then Lubich [4] considered the behavior of the solution near 0 . He showed that, if $f$ is analytic at the origin, then there exists a function $Y$ of two variables that is analytic at the origin such that

$$
y(x)=Y\left(x, x^{\alpha}\right), \quad 0 \leqslant x \leqslant h,
$$

for some $0<h \leqslant a$. The above work suggests that non-smoothness of the solution to (1.1) is generally unavoidable. However, Diethelm [1] established a sufficient and necessary condition under which $y$ is analytic on $[0, h]$ for some $0<h \leqslant a$. But, since we have already seen that non-smoothness of $y$ is generally unavoidable, it is not surprising that this condition is unrealistic. Recently, Deng [3] proposed two conditions: under the first condition the solution belongs to $C^{m}$ for some positive integer $m$; under the second one the solution is a polynomial. It should be noted that, the second condition is just the one proposed in [1], and the first condition is also unrealistic.

The main result of this paper is that, although the solution $y$ of (1.1) does not generally belong to $C^{m}$ for some positive integer $m$, we can still construct a non-smooth function of the form

$$
S(x):=c_{0}+\sum_{j=1}^{n} c_{j} x^{\gamma_{j}}
$$

such that

$$
y-S \in C^{m},
$$

provided $f$ is sufficiently smooth. Most importantly, given $c_{0}$ and $f$, we can obtain $S$ by a simple computation. This is significant in the development of numerical methods for (1.1). In addition, we obtain a sufficient and necessary condition under which $y \in C^{m}$. We note that this condition is essentially the same as the first condition mentioned already in [3, Theorem 2.8], but the necessity was not considered therein.

The rest of this paper is organized as follows. In Section 2 we introduce some basic notation and preliminaries. In Section 3 we state the main results of this paper, and present their proofs in Section 4.

\section{Notation and Preliminaries}

Let $0<h<\infty$. We use $C[0, h]$ to denote the space of all continuous real functions defined on $[0, h]$. For any $k \in \mathbb{N}_{>0}$ and $0 \leqslant \gamma \leqslant 1$, define

$$
\begin{aligned}
C^{k}[0, h] & :=\left\{v \in C[0, h]: v^{(j)} \in C[0, h] \quad \text { for } j=1,2, \ldots, k\right\}, \\
C^{k, \gamma}[0, h] & :=\left\{v \in C^{k}[0, h]: \max _{0 \leqslant x<y \leqslant h}|v|_{C^{k, \gamma}[0, h]}<\infty\right\},
\end{aligned}
$$

and endow the above two spaces with two norms respectively by

$$
\begin{aligned}
\|v\|_{C^{k}[0, h]}:=\max _{0 \leqslant j \leqslant k} \max _{0 \leqslant x \leqslant h}\left|v^{(j)}(x)\right| & \text { for all } v \in C^{k}[0, h], \\
\|v\|_{C^{k, \gamma}[0, h]}:=\max \left\{\|v\|_{C^{k}[0, h]},|v|_{C^{k, \gamma}[0, h]}\right\} & \text { for all } v \in C^{k, \gamma}[0, h] .
\end{aligned}
$$

Here the semi-norm $|\cdot|_{C^{k, \gamma}[0, h]}$ is given by

$$
|v|_{C^{k, \gamma}[0, h]}:=\sup _{0 \leqslant x<y \leqslant h} \frac{\left|v^{(k)}(x)-v^{(k)}(y)\right|}{(y-x)^{\gamma}}
$$

for all $v \in C^{k, \gamma}[0, h]$, and it is obvious that $C^{k}[0, h]$ coincides with $C^{k, 0}[0, h]$.

For any $s \in \mathbb{N}_{>0}$, define

$$
\Lambda_{s}:=\left\{\beta=\left(\beta_{1}, \beta_{2}, \ldots, \beta_{s}\right) \in\{1,2\}^{s}\right\},
$$

and, for any $\beta \in \Lambda_{s}$, we use the following notation:

$$
\partial_{\beta} g:=\frac{\partial}{\partial x_{\beta_{s}}} \frac{\partial}{\partial x_{\beta_{s-1}}} \cdots \frac{\partial}{\partial x_{\beta_{1}}} g\left(x_{1}, x_{2}\right),
$$


where $g$ is a real function of two variables. In addition, we define

$$
\Lambda_{0}:=\{\emptyset\},
$$

and denote by $\partial_{\emptyset}$ the identity mapping.

\section{Main Results}

Let us first make the following assumption on $f$.

Assumption 1. There exist a positive integer n, and a positive constant $M$ such that

$$
\begin{array}{r}
f \in C^{n}\left([0, a] \times\left[c_{0}-b, c_{0}+b\right]\right), \\
\max _{(x, y) \in[0, a] \times\left[c_{0}-b, c_{0}+b\right]} \max _{\substack{0 \leqslant i \leqslant n \\
0 \leqslant j \leqslant n \\
i+j \leqslant n}}\left|\frac{\partial^{i}}{\partial x^{i}} \frac{\partial^{j}}{\partial y^{j}} f(x, y)\right| \leqslant M .
\end{array}
$$

Throughout this paper, we assume that the above assumption is fulfilled.

Define $J \in \mathbb{N}$ and a strictly increasing sequence $\left\{\gamma_{i}\right\}_{i=1}^{J}$ by

$$
\left\{\gamma_{j}: 1 \leqslant j \leqslant J\right\}=\{i+j \alpha: i, j \in \mathbb{N}, 0<i+j \alpha<m\},
$$

where

$$
m:=\max \{j \in \mathbb{N}: j<n \alpha\}
$$

Define $c_{1}, c_{2}, \ldots, c_{J} \in \mathbb{R}$ by

$$
Q(x)-S(x)+c_{0} \in \operatorname{span}\left\{x^{i+j \alpha}: i, j \in \mathbb{N}, i+j \alpha \geqslant m\right\},
$$

where

$$
Q(x):=\sum_{s=0}^{n-1} \sum_{\beta \in \Lambda_{s}} \frac{\partial_{\beta} f\left(0, c_{0}\right)}{\Gamma(\alpha)} \int_{0}^{x}\left(x-t_{0}\right)^{\alpha-1} \mathrm{~d} t_{0} \prod_{k=1}^{s} \int_{0}^{t_{k-1}} \frac{1+(-1)^{\beta_{k}+1}}{2}+\frac{1+(-1)^{\beta_{k}}}{2} \sum_{j=1}^{J} \gamma_{j} c_{j} t_{k}^{\gamma_{j}-1} \mathrm{~d} t_{k},
$$

and

$$
S(x):=c_{0}+\sum_{j=1}^{J} c_{j} x^{\gamma_{j}} .
$$

Above and throughout, a product of a sequence of integrals should be understood in expanded form. For example, (3.4) is understood by

$$
\begin{aligned}
Q(x):=\sum_{s=0}^{n-1} \sum_{\beta \in \Lambda_{s}} \frac{\partial_{\beta} f\left(0, c_{0}\right)}{\Gamma(\alpha)} \int_{0}^{x}\left(x-t_{0}\right)^{\alpha-1} \mathrm{~d} t_{0} & \int_{0}^{t_{0}} \frac{1+(-1)^{\beta_{1}+1}}{2}+\frac{1+(-1)^{\beta_{1}}}{2} \sum_{j=1}^{J} \gamma_{j} c_{j} t_{1}^{\gamma_{j}-1} \mathrm{~d} t_{1} \\
& \int_{0}^{t_{1}} \frac{1+(-1)^{\beta_{2}+1}}{2}+\frac{1+(-1)^{\beta_{2}}}{2} \sum_{j=1}^{J} \gamma_{j} c_{j} t_{2}^{\gamma_{j}-1} \mathrm{~d} t_{2} \\
& \cdots \\
& \int_{0}^{t_{s-1}} \frac{1+(-1)^{\beta_{s}+1}}{2}+\frac{1+(-1)^{\beta_{s}}}{2} \sum_{j=1}^{J} \gamma_{j} c_{j} t_{s}^{\gamma_{j}-1} \mathrm{~d} t_{s} .
\end{aligned}
$$

Remark 3.1. It is easy to see that we can express $Q$ in the form

$$
Q(x)=\sum_{j=1}^{L} d_{j} x^{\gamma_{j}},
$$

where $\left\{\gamma_{j}\right\}_{j=J+1}^{L}$ is a strictly increasing sequence such that $\gamma_{J}<\gamma_{J+1}$ and

$$
\left\{\gamma_{j}: 1 \leqslant j \leqslant L\right\}=\left\{i+j \alpha: i, j \in \mathbb{N}, i \leqslant n-1,1 \leqslant j \leqslant 1+(n-1) \gamma_{J}\right\} .
$$


Moreover, for $1 \leqslant j \leqslant J$, the value of $d_{j}$ only depends on $c_{0}, c_{1}, \ldots, c_{j-1}$, and $f$ (more precisely, $\left.\partial_{\beta} f\left(0, c_{0}\right), \beta \in \Lambda_{s}, 1 \leqslant s \leqslant n-1\right)$. Obviously, there exist(s) uniquely $c_{1}, c_{2}, \ldots, c_{J}$ such that (3.3) holds, and hence $c_{1}, c_{2}, \ldots, c_{J}$ are/is well-defined. Furthermore, if $\gamma_{J}+\alpha-m>0$, then

$$
Q-S \in C^{m, \gamma_{J}+\alpha-m}[0, a]
$$

and if $\gamma_{J}+\alpha-m=0$, then

$$
Q-S \in C^{m, \alpha}[0, a]
$$

Remark 3.2. Note that, $S$ only depends on $c_{0}$ and

$$
\left\{\partial_{\beta} f\left(0, c_{0}\right): \beta \in \Lambda_{s}, 0 \leqslant s<n\right\} .
$$

Since $c_{0}$ and $f$ are already available, we can obtain $S$ by a simple calculation.

Define

$$
h^{*}:=\min \left\{a,\left(\frac{b \Gamma(1+\alpha)}{M}\right)^{\frac{1}{\alpha}}\right\} .
$$

By [2, Theorem 2.2] we know that there exists a unique solution $y^{*} \in C\left[0, h^{*}\right]$ to (1.1). Now we state the most important result of this paper in the following theorem.

Theorem 3.1. There exist two positive constant $C_{0}$ and $C_{1}$ that only depends on a, $\alpha$ and $M$, such that, for any $0<h \leqslant h^{*}$ and $K>0$ such that

$$
\left\|(Q-S)^{\prime}\right\|_{C^{m-1}[0, h]}+C_{1} h^{\alpha}+C_{0} h^{\alpha} \sum_{j=1}^{m} K^{j} \leqslant K,
$$

we have $y^{*}-S \in C^{m}[0, h]$ and

$$
\left\|\left(y^{*}-S\right)^{\prime}\right\|_{C^{m-1}[0, h]} \leqslant K .
$$

Corollary 3.1. There exists $0<h \leqslant h^{*}$ such that $y^{*} \in C^{m}[0, h]$ if, and only if,

$$
\frac{\partial^{i}}{\partial x^{i}} f\left(0, c_{0}\right)=0 \quad \text { for all } 0 \leqslant i<m .
$$

Remark 3.3. Corollary 3.1 states that $y^{*} \in C^{1}[0, h]$ for some $0<h \leqslant h^{*}$ if and only if $f\left(0, c_{0}\right)=0$. So we only have $y^{*} \in C[0, h] \backslash C^{1}[0, h]$, if $f\left(0, c_{0}\right) \neq 0$. This yields great difficulty in developing high order numerical methods for (1.1), although $y^{*} \in C^{m}(0, h]$. Many numerical methods for (1.1) may not even converge theoretically, since they require that $y^{*} \in C^{m}[0, h]$ for some positive integer $m$. However, we can obtain the numerical values of $y^{*}$ at some left-most nodes by solving the following problem $\left(y^{*}=y+S\right)$ : seek $y \in C^{m}[0, \tilde{h}]$ such that

$$
y(x)=c_{0}-S(x)+\frac{1}{\Gamma(\alpha)} \int_{0}^{x}(x-t)^{\alpha-1} f(t, y(t)+S(t)) \mathrm{d} t, \quad 0 \leqslant x \leqslant \widetilde{h},
$$

where $\widetilde{h} \ll h$. Then we start the numerical methods for (1.1).

Remark 3.4. Assuming that $f$ satisfies $f\left(x, c_{0}\right)=0$ for all $0 \leqslant x \leqslant a$, it is easy to see that

$$
c_{i}=0 \quad \text { for all } 1 \leqslant i \leqslant J
$$

and hence $S=c_{0}$. Then Theorem 3.1 implies $y^{*} \in C^{m}[0, h]$. Actually, in this case, it is easy to see that $y^{*}=c_{0}$.

Remark 3.5. Put

$$
\Theta:=\left\{1 \leqslant j \leqslant J: \gamma_{j} \notin \mathbb{N}\right\}
$$

Obviously,

$$
\sum_{j \in \Theta} c_{j} x^{\gamma_{j}}
$$

is the singular part (compared to the $C^{m}$ regularity) in $S$, and thus the singular part in $y^{*}$. Corollary 3.1 essentially claims that (3.7) holds if and only if $c_{j}=0$ for all $j \in \Theta$. Since (3.7) is rare, we can consider singularity as an intrinsic property of solutions to fractional differential equations. In addition, we have the following result: that $c_{j}=0$ for all $1 \leqslant j \leqslant J$ is equivalent to that $c_{j}=0$ for all $j \in \Theta$. This is contained in the proof of Corollary 3.1 in Section 4.3. 


\section{Proofs}

Let $0<h<\infty$. For any $k \in \mathbb{N}$ and $\gamma \in[0,1]$, define

$$
\begin{aligned}
& \mathcal{C}^{k, \gamma}[0, h]:=\left\{v \in C^{k, \gamma}[0, h]: v^{(j)}(0)=0, \quad j=0,1,2, \ldots, k\right\}, \\
& \widehat{\mathcal{C}}^{k, \gamma}[0, h]:=\left\{v \in \mathcal{C}^{k, \gamma}[0, h]:\left\|v+S-c_{0}\right\|_{C[0, h]} \leqslant b\right\} .
\end{aligned}
$$

In particular, we use $\mathcal{C}^{k}[0, h]$ and $\widehat{\mathcal{C}}^{k}[0, h]$ to abbreviate $\mathcal{C}^{k, 0}[0, h]$ and $\widehat{\mathcal{C}}^{k, 0}[0, h]$ respectively for $k \in \mathbb{N}_{>0}$, and use $\mathcal{C}[0, h]$ and $\widehat{\mathcal{C}}[0, h]$ to abbreviate $\mathcal{C}^{0}[0, h]$ and $\widehat{\mathcal{C}}^{0}[0, h]$ respectively. In addition, for a function $v$ defined on $(0, h]$ with $h>0$, by $v \in \mathcal{C}^{k, \gamma}[0, h]$ we mean that, setting $v(0):=0$, the function $v$ belongs to $\mathcal{C}^{k, \gamma}[0, h]$.

In the remainder of this paper, unless otherwise specified, we use $C$ to denote a positive constant that only depends on $\alpha, a$ and $M$, and its value may differ at each occurrence. By the definitions of $c_{1}, c_{2}$, $\ldots, c_{J}$, it is easy to see that $\left|c_{j}\right| \leqslant C$ for all $1 \leqslant j \leqslant J$, and we use this implicitly in the forthcoming analysis.

\subsection{Some Auxiliary Results}

We start by introducing some operators. For $0<h \leqslant a$, define $\mathcal{P}_{1, h}: \widehat{\mathcal{C}}^{m}[0, h] \rightarrow \mathcal{C}[0, h], \mathcal{P}_{2, h}: \widehat{\mathcal{C}}^{m}[0, h] \rightarrow$ $\mathcal{C}[0, h]$, and $\mathcal{P}_{3, h}: \widehat{\mathcal{C}}^{m}[0, h] \rightarrow \mathcal{C}[0, h]$, respectively, by

$$
\begin{aligned}
& \mathcal{P}_{1, h} z(x):=\frac{1}{\Gamma(\alpha)} \int_{0}^{x}(x-t)^{\alpha-1} \mathcal{G}_{1, h} z(t) \mathrm{d} t, \\
& \mathcal{P}_{2, h} z(x):=\frac{1}{\Gamma(\alpha)} \int_{0}^{x}(x-t)^{\alpha-1} \mathcal{G}_{2, h} z(t) \mathrm{d} t, \\
& \mathcal{P}_{3, h} z(x):=\frac{1}{\Gamma(\alpha)} \int_{0}^{x}(x-t)^{\alpha-1} \mathcal{G}_{3, h} z(t) \mathrm{d} t,
\end{aligned}
$$

for all $z \in \widehat{\mathcal{C}}^{m}[0, h]$, where $\mathcal{G}_{1, h} z, \mathcal{G}_{2, h} z, \mathcal{G}_{3, h} z \in \mathcal{C}[0, h]$ are given respectively by

$$
\begin{aligned}
& \mathcal{G}_{1, h} z\left(t_{0}\right):=\sum_{s=1}^{n} \sum_{\substack{\beta \in \Lambda_{s} \\
\beta_{s}=2}} \prod_{k=1}^{s-1} \int_{0}^{t_{k-1}} \frac{1+(-1)^{\beta_{k}+1}}{2}+\frac{1+(-1)^{\beta_{k}}}{2} \sum_{j=1}^{J} \gamma_{j} c_{j} t_{k}^{\gamma_{j}-1} \mathrm{~d} t_{k} \\
& \int_{0}^{t_{s-1}} z^{\prime}\left(t_{s}\right) \partial_{\beta} f\left(t_{s}, z\left(t_{s}\right)+S\left(t_{s}\right)\right) \mathrm{d} t_{s} \\
& \mathcal{G}_{2, h} z\left(t_{0}\right):=\sum_{\substack{\beta \in \Lambda_{n} \\
\beta_{n}=2}} \prod_{k=1}^{n-1} \int_{0}^{t_{k-1}} \frac{1+(-1)^{\beta_{k}+1}}{2}+\frac{1+(-1)^{\beta_{k}}}{2} \sum_{j=1}^{J} \gamma_{j} c_{j} t_{k}^{\gamma_{j}-1} \mathrm{~d} t_{k} \\
& \int_{0}^{t_{n-1}} \partial_{\beta} f\left(t_{n}, z\left(t_{n}\right)+S\left(t_{n}\right)\right) \sum_{j=1}^{J} \gamma_{j} c_{j} t_{n}^{\gamma_{j}-1} \mathrm{~d} t_{n} \\
& \mathcal{G}_{3, h} z\left(t_{0}\right):=\sum_{\substack{\beta \in \Lambda_{n} \\
\beta_{n}=1}} \prod_{k=1}^{n-1} \int_{0}^{t_{k-1}} \frac{1+(-1)^{\beta_{k}+1}}{2}+\frac{1+(-1)^{\beta_{k}}}{2} \sum_{j=1}^{J} \gamma_{j} c_{j} t_{k}^{\gamma_{j}-1} \mathrm{~d} t_{k} \\
& \int_{0}^{t_{n-1}} \partial_{\beta} f\left(t_{n}, z\left(t_{n}\right)+S\left(t_{n}\right)\right) \mathrm{d} t_{n}
\end{aligned}
$$

for all $0 \leqslant t_{0} \leqslant h$.

Then let us present the following important results for the above operators.

Lemma 4.1. Let $0<h \leqslant a$. For any $z \in \widehat{\mathcal{C}}^{m}[0, h]$, we have

$$
\frac{1}{\Gamma(\alpha)} \int_{0}^{x}(x-t)^{\alpha-1} f(t, z(t)+S(t)) \mathrm{d} t=Q(x)+\mathcal{P}_{1, h} z(x)+\mathcal{P}_{2, h} z(x)+\mathcal{P}_{3, h} z(x)
$$

for all $0 \leqslant x \leqslant h$. 
Proof. Let $\beta \in \Lambda_{s}$ with $1 \leqslant s<n$. For any $0<t_{s} \leqslant h$, applying the fundamental theorem of calculus yields

$$
\begin{aligned}
\partial_{\beta} f\left(t_{s}, z\left(t_{s}\right)+S\left(t_{s}\right)\right)= & \partial_{\beta} f(\epsilon, z(\epsilon)+S(\epsilon))+\int_{\epsilon}^{t_{s}} \partial_{\widetilde{\beta}} f\left(t_{s+1}, z\left(t_{s+1}\right)+S\left(t_{s+1}\right)\right) \mathrm{d} t_{s+1}+ \\
& \int_{\epsilon}^{t_{s}}\left(z^{\prime}\left(t_{s+1}\right)+\sum_{j=1}^{J} \gamma_{j} c_{j} t_{s+1}^{\gamma_{j}-1}\right) \partial_{\widetilde{\beta}} f\left(t_{s+1}, z\left(t_{s+1}\right)+S\left(t_{s+1}\right)\right) \mathrm{d} t_{s+1}
\end{aligned}
$$

for all $0<\epsilon \leqslant t_{s}$, where $\widetilde{\beta}:=\left(\beta_{1}, \beta_{2}, \ldots, \beta_{s}, 1\right)$ and $\widetilde{\widetilde{\beta}}:=\left(\beta_{1}, \beta_{2}, \ldots, \beta_{s}, 2\right)$. Taking limits on both sides of the above equation as $\epsilon$ approaches $0+$, we obtain

$$
\begin{aligned}
\partial_{\beta} f\left(t_{s}, z\left(t_{s}\right)+S\left(t_{s}\right)\right)= & \left.\partial_{\beta} f\left(0, c_{0}\right)\right)+\int_{0}^{t_{s}} \partial_{\widetilde{\beta}} f\left(t_{s+1}, z\left(t_{s+1}\right)+S\left(t_{s+1}\right)\right) \mathrm{d} t_{s+1}+ \\
& \int_{0}^{t_{s}}\left(z^{\prime}\left(t_{s+1}\right)+\sum_{j=1}^{J} \gamma_{j} c_{j} t_{s+1}^{\gamma_{j}-1}\right) \partial_{\widetilde{\beta}} f\left(t_{s+1}, z\left(t_{s+1}\right)+S\left(t_{s+1}\right)\right) \mathrm{d} t_{s+1} .
\end{aligned}
$$

Using this equality repeatedly, we easily obtain (4.9). This completes the proof.

Lemma 4.2. Let $0<h \leqslant a$. For any $z \in \widehat{\mathcal{C}}^{m}[0, h]$, we have $\mathcal{P}_{1, h} z \in \mathcal{C}^{m, \alpha}[0, h]$ and

$$
\begin{gathered}
\left\|\left(\mathcal{P}_{1, h} z\right)^{\prime}\right\|_{C^{m-1}[0, h]} \leqslant C h^{\alpha} \sum_{j=1}^{m}\left\|z^{\prime}\right\|_{C^{m-1}[0, h]}^{j}, \\
\left|\left(\mathcal{P}_{1, h} z\right)^{(m)}\right|_{C^{0, \alpha}[0, h]} \leqslant C \sum_{j=1}^{m}\left\|z^{\prime}\right\|_{C^{m-1}[0, h]}^{j} .
\end{gathered}
$$

Lemma 4.3. Let $0<h \leqslant a$. For any $z \in \widehat{\mathcal{C}}^{m}[0, h]$, we have $\mathcal{P}_{2, h} z, \mathcal{P}_{3, h} z \in \mathcal{C}^{m, \alpha}[0, h]$ and

$$
\begin{gathered}
\left\|\left(\mathcal{P}_{2, h} z\right)^{\prime}\right\|_{C^{m-1}[0, h]}+\left\|\left(\mathcal{P}_{3, h} z\right)^{\prime}\right\|_{C^{m-1}[0, h]} \leqslant C h^{\alpha}, \\
\left|\left(\mathcal{P}_{2, h} z\right)^{(m)}\right|_{C^{0, \alpha}[0, h]}+\left|\left(\mathcal{P}_{3, h} z\right)^{(m)}\right|_{C^{0, \alpha}[0, h]} \leqslant C .
\end{gathered}
$$

To prove the above two lemmas, we need several lemmas below.

Lemma 4.4. Let $0<h \leqslant a$ and $g \in \mathcal{C}^{m}[0, h]$. We have $w \in \mathcal{C}^{m, \alpha}[0, h]$ and

$$
\begin{aligned}
\left\|w^{\prime}\right\|_{C^{m-1}[0, h]} & \leqslant C h^{\alpha}\left\|g^{\prime}\right\|_{C^{m-1}[0, h]}, \\
\left|w^{(m)}\right|_{C^{0, \alpha}[0, h]} & \leqslant C\left\|g^{(m)}\right\|_{C[0, h]},
\end{aligned}
$$

where

$$
w(x):=\int_{0}^{x}(x-t)^{\alpha-1} g(t) \mathrm{d} t, \quad 0 \leqslant x \leqslant h .
$$

Proof. Since $g \in \mathcal{C}^{m}[0, h]$ we have

$$
w^{(i)}(x)=\int_{0}^{x}(x-t)^{\alpha-1} g^{(i)}(t) \mathrm{d} t, \quad 1 \leqslant i \leqslant m .
$$

Then $w \in \mathcal{C}^{m}[0, h]$ and (4.14) follow, and (4.15) follows from [6, Theorem 3.1]. This completes the proof.

Lemma 4.5. Let $0<h \leqslant a$, and $k, l \in \mathbb{N}$ such that $k \leqslant m$ and $l \alpha \leqslant 1$. For any $g \in \mathcal{C}^{k, l \alpha}[0, h]$, define

$$
w(x):=\int_{0}^{x} \sum_{j=1}^{J} \gamma_{j} c_{j} t^{\gamma_{j}-1} g(t) \mathrm{d} t, \quad 0<x \leqslant h .
$$

Then we have the following results: 
- If $(l+1) \alpha \leqslant 1$, then we have $w \in \mathcal{C}^{k,(l+1) \alpha}[0, a]$ and

$$
\|w\|_{\mathcal{C}^{k,(l+1) \alpha}} \leqslant C\|g\|_{\mathcal{C}^{k, l \alpha}} .
$$

- If $(l+1) \alpha>1$, then we have $w \in \mathcal{C}^{k+1,(l+1) \alpha-1}[0, a]$ and

$$
\|w\|_{\mathcal{C}^{k+1,(l+1) \alpha-1}} \leqslant C\|g\|_{\mathcal{C}^{k, l \alpha}} .
$$

For any $0<h \leqslant a, w \in \mathcal{C}[0, h]$, and $\beta \in \Lambda_{s}$ with $1 \leqslant s \leqslant n$, define $\mathcal{T}_{w, \beta, h}: \widehat{\mathcal{C}}^{m}[0, h] \rightarrow \mathcal{C}[0, h]$ by

$$
\mathcal{T}_{w, \beta, h} z(x):=w(x) \partial_{\beta} f(x, z(x)+S(x)),
$$

for all $z \in \widehat{\mathcal{C}}^{m}[0, h]$.

Lemma 4.6. For $0 \leqslant k \leqslant m$, we have $\mathcal{T}_{w, \beta, h} z \in \mathcal{C}^{\min \{k, n-s\}}[0, h]$ and

$$
\left\|\mathcal{T}_{w, \beta, h} z\right\|_{C^{\min \{k, n-s\}}[0, h]} \leqslant C\|w\|_{C^{k}[0, h]} \sum_{j=0}^{\min \{k, n-s\}}\left\|z^{\prime}\right\|_{C^{m-1}[0, h]}^{j}
$$

for all $0<h \leqslant a, w \in \mathcal{C}^{k}[0, h], \beta \in \Lambda_{s}$ with $1 \leqslant s \leqslant n$, and $z \in \widehat{\mathcal{C}}^{m}[0, h]$.

The proofs of Lemmas 4.5 and 4.6 are presented in Appendix A. In the rest of this subsection, we give the proofs of Lemmas 4.2 and 4.3 .

Proof of Lemma 4.2. By (4.3), (4.6), and Lemma 4.4, it suffices to show that, for each $\beta \in \Lambda_{s}$ with $\beta_{s}=2$, we have $g_{0} \in \mathcal{C}^{m}[0, h]$ and

$$
\left\|g_{0}\right\|_{C^{m}[0, h]} \leqslant C \sum_{j=1}^{\min \{m, n-s+1\}}\left\|z^{\prime}\right\|_{C^{m-1}[0, h]}^{j},
$$

where, if $s=1$, then

$$
g_{0}(x):=\int_{0}^{x} z^{\prime}(t) \partial_{2} f(t, z(t)+S(t)) \mathrm{d} t
$$

if $2 \leqslant s \leqslant n$, then

$$
\begin{aligned}
g_{0}(x) & :=\int_{0}^{x}\left(\frac{1+(-1)^{\beta_{1}+1}}{2}+\frac{1+(-1)^{\beta_{1}}}{2} \sum_{j=1}^{J} \gamma_{j} c_{j} t^{\gamma_{j}-1}\right) g_{1}(t) \mathrm{d} t, \\
g_{1}(x) & :=\int_{0}^{x}\left(\frac{1+(-1)^{\beta_{2}+1}}{2}+\frac{1+(-1)^{\beta_{2}}}{2} \sum_{j=1}^{J} \gamma_{j} c_{j} t^{\gamma_{j}-1}\right) g_{2}(t) \mathrm{d} t, \\
& \vdots \\
g_{s-2}(x) & :=\int_{0}^{x}\left(\frac{1+(-1)^{\beta_{s-1}+1}}{2}+\frac{1+(-1)^{\beta_{s-1}}}{2} \sum_{j=1}^{J} \gamma_{j} c_{j} t^{\gamma_{j}-1}\right) g_{s-1}(t) \mathrm{d} t, \\
g_{s-1}(x) & :=\int_{0}^{x} z^{\prime}(t) \partial_{\beta} f(t, z(t)+S(t)) \mathrm{d} t .
\end{aligned}
$$

To do so, we proceed as follows. If $s=1$, then by Lemma 4.6 we obtain $g_{0} \in \mathcal{C}^{m}[0, h]$ and (4.17). Let us suppose that $2 \leqslant s \leqslant n$. By Lemma 4.6 it follows $g_{s-1} \in \mathcal{C}^{\min \{m, n-s+1\}}[0, h]$ and

$$
\left\|g_{s-1}\right\|_{C^{\min \{m, n-s+1\}}[0, h]} \leqslant C \sum_{j=1}^{\min \{m, n-s+1\}}\left\|z^{\prime}\right\|_{C^{m-1}[0, h]}^{j} .
$$

Then, by the simple estimate

$$
(n-s+1)+(s-1) \alpha>m,
$$


applying Lemma 4.5 to $g_{s-2}, g_{s-3}, \ldots, g_{0}$ successively yields $g_{0} \in \mathcal{C}^{m}[0, h]$ and (4.17). This completes the proof of Lemma 4.2.

Proof of Lemma 4.3. Let us first show that $\mathcal{G}_{2, h} z \in \mathcal{C}^{m}[0, h]$ and

$$
\left\|\mathcal{G}_{2, h} z\right\|_{C^{m}[0, h]} \leqslant C .
$$

By (4.7) it suffices to show that, for any $\beta \in \Lambda_{n}$ with $\beta_{n}=2$, we have $g_{0} \in \mathcal{C}^{m}[0, h]$ and

$$
\left\|g_{0}\right\|_{C^{m}[0, h]} \leqslant C
$$

where

$$
\begin{aligned}
g_{0}(x) & :=\int_{0}^{x}\left(\frac{1+(-1)^{\beta_{1}+1}}{2}+\frac{1+(-1)^{\beta_{1}}}{2} \sum_{j=1}^{J} \gamma_{j} c_{j} t^{\gamma_{j}-1}\right) g_{1}(t) \mathrm{d} t, \\
g_{1}(x) & :=\int_{0}^{x}\left(\frac{1+(-1)^{\beta_{2}+1}}{2}+\frac{1+(-1)^{\beta_{2}}}{2} \sum_{j=1}^{J} \gamma_{j} c_{j} t^{\gamma_{j}-1}\right) g_{2}(t) \mathrm{d} t, \\
& \vdots \\
g_{n-2}(x) & :=\int_{0}^{x}\left(\frac{1+(-1)^{\beta_{s-1}+1}}{2}+\frac{1+(-1)^{\beta_{s-1}}}{2} \sum_{j=1}^{J} \gamma_{j} c_{j} t^{\gamma_{j}-1}\right) g_{s-1}(t) \mathrm{d} t, \\
g_{n-1}(x) & :=\int_{0}^{x} \partial_{\beta} f(t, z(t)+S(t)) \sum_{j=1}^{J} \gamma_{j} c_{j} t^{\gamma_{j}-1} \mathrm{~d} t
\end{aligned}
$$

for all $0 \leqslant x \leqslant h$. Noting the fact that

$$
\partial_{\beta} f(\cdot, z(\cdot)+S(\cdot)) \in C[0, h]
$$

and $\gamma_{j} \geqslant \alpha$ for all $1 \leqslant j \leqslant J$, we easily obtain $g_{n-1} \in \mathcal{C}^{0, \alpha}[0, h]$ and

$$
\left\|g_{n-1}\right\|_{C^{0, \alpha}[0, h]} \leqslant C \text {. }
$$

Then, applying Lemma 4.5 to $g_{n-2}, g_{n-3}, \ldots, g_{0}$ successively, and using the fact $n \alpha>m$, we obtain $g_{0} \in \mathcal{C}^{m}[0, h]$ and (4.19). Thus we have showed $\mathcal{G}_{2, h} z \in \mathcal{C}^{m}[0, h]$ and (4.18).

Similarly, we can show that $\mathcal{G}_{3, h} z \in \mathcal{C}^{m}[0, h]$ and $\left\|\mathcal{G}_{3, h} z\right\|_{C^{m}[0, h]} \leqslant C$. Consequently, by (4.4), (4.5), and Lemma 4.4, we infer that $\mathcal{P}_{2, h} z, \mathcal{P}_{3, h} z \in \mathcal{C}^{m, \alpha}[0, h]$, and (4.12) and (4.13) hold. This completes the proof.

\subsection{Proof of Theorem 3.1}

By Lemmas 4.2 and 4.3 there exist two positive constants $C_{0}$ and $C_{1}$ that only depend on $a, \alpha$ and $M$, such that

$$
\begin{gathered}
\left\|\left(\mathcal{P}_{1, h} z\right)^{\prime}\right\|_{C^{m-1}[0, h]} \leqslant C_{0} h^{\alpha} \sum_{j=1}^{m}\left\|z^{\prime}\right\|_{C^{m-1}[0, h]}^{j}, \\
\left\|\left(\mathcal{P}_{2, h} z\right)^{\prime}\right\|_{C^{m-1}[0, h]}+\left\|\left(\mathcal{P}_{3, h} z\right)^{\prime}\right\|_{C^{m-1}[0, h]} \leqslant C_{1} h^{\alpha},
\end{gathered}
$$

for all $0<h \leqslant a$ and $z \in \widehat{\mathcal{C}}^{m}[0, h]$. Let $0<h \leqslant h^{*}$ and $K>0$ such that

$$
\left\|(Q-S)^{\prime}\right\|_{C^{m-1}[0, h]}+C_{1} h^{\alpha}+C_{0} h^{\alpha} \sum_{j=1}^{m} K^{j} \leqslant K .
$$

Define $\mathcal{J}: V \rightarrow \mathcal{C}[0, h]$ by

$$
\mathcal{J} z(x):=c_{0}-S(x)+\frac{1}{\Gamma(\alpha)} \int_{0}^{x}(x-t)^{\alpha-1} f(t, z(t)+S(t)) \mathrm{d} t,
$$

for all $z \in V$ and $x \in[0, h]$, where

$$
V:=\left\{v \in \widehat{\mathcal{C}}^{m}[0, h]:\left\|v^{\prime}\right\|_{C^{m-1}[0, h]} \leqslant K\right\} .
$$


Remark 4.1. It is clear that $V$ is a bounded, closed, convex subset of $C^{m}[0, h]$.

Remark 4.2. Let $\delta>0$. If we put

$$
\begin{aligned}
& K:=\left\|(Q-S)^{\prime}\right\|_{C^{m-1}[0, h]}+C_{1} a^{\alpha}+\delta, \\
& h:=\min \left\{h^{*},\left(\delta^{-1} C_{0} \sum_{j=1}^{m} K^{j}\right)^{-\frac{1}{\alpha}}\right\},
\end{aligned}
$$

then (4.22) holds.

For the operator $\mathcal{J}$, we have the following key result.

Lemma 4.7. For each $z \in V$, we have $\mathcal{J} z \in V$ and

$$
\left|(\mathcal{J} z)^{(m)}\right|_{C^{0, \gamma}[0, h]} \leqslant\left|(Q-S)^{(m)}\right|_{C^{0, \gamma}[0, h]}+C \sum_{j=0}^{m} K^{j}
$$

where $\gamma:=\alpha$ if $\gamma_{J}+\alpha=m$, and $\gamma:=\gamma_{J}+\alpha-m$ if $\gamma_{J}+\alpha>m$.

Proof. Let us first show $\mathcal{J} z \in V$. Using (4.23) and the fact $h \leqslant\left(\frac{b \Gamma(1+\alpha)}{M}\right)^{\frac{1}{\alpha}}$, we have

$$
\left|\mathcal{J} z(x)+S(x)-c_{0}\right|=\frac{1}{\Gamma(\alpha)}\left|\int_{0}^{x}(x-t)^{\alpha-1} f(t, z(t)+S(t)) \mathrm{d} t\right| \leqslant \frac{M h^{\alpha}}{\Gamma(1+\alpha)} \leqslant b
$$

for all $x \in[0, h]$, and so

$$
\left\|\mathcal{J} z+S-c_{0}\right\|_{C[0, h]} \leqslant b
$$

By Lemma 4.1 we have

$$
\mathcal{J} z(x)=c_{0}-S(x)+Q(x)+\mathcal{P}_{1, h} z(x)+\mathcal{P}_{2, h} z(x)+\mathcal{P}_{3, h} z(x),
$$

and then, by Lemmas 4.2 and 4.3 , and the fact $c_{0}-S+Q \in \mathcal{C}^{m}[0, h]$, we obtain $\mathcal{J} z \in \mathcal{C}^{m}[0, h]$. It remains, therefore, to show that

$$
\left\|(\mathcal{J} z)^{\prime}\right\|_{C^{m-1}[0, h]} \leqslant K .
$$

To this end, note that, by (4.26), (4.20) and (4.21) we obtain

$$
\left\|(\mathcal{J} z)^{\prime}\right\|_{C^{m-1}[0, h]} \leqslant\left\|(Q-S)^{\prime}\right\|_{C^{m-1}[0, h]}+C_{1} h^{\alpha}+C_{0} h^{\alpha} \sum_{j=1}^{m} K^{j},
$$

and then (4.27) follows from (4.22). We have thus showed $\mathcal{J} z \in V$.

Finally, let us show (4.25). By Lemmas 4.2 and 4.3 we obtain

$$
\left|\left(\mathcal{P}_{1, h} z\right)^{(m)}\right|_{C^{0, \alpha}[0, h]}+\left|\left(\mathcal{P}_{2, h} z\right)^{(m)}\right|_{C^{0, \alpha}[0, h]}+\left|\left(\mathcal{P}_{3, h} z\right)^{(m)}\right|_{C^{0, \alpha}[0, h]} \leqslant C \sum_{j=0}^{m}\left\|z^{\prime}\right\|_{C^{m-1}[0, h]}^{j} \leqslant C \sum_{j=0}^{m} K^{j} .
$$

From the fact $\gamma \leqslant \alpha$ it follows

$$
\left|\left(\mathcal{P}_{1, h} z\right)^{(m)}\right|_{C^{0, \gamma}[0, h]}+\left|\left(\mathcal{P}_{2, h} z\right)^{(m)}\right|_{C^{0, \gamma}[0, h]}+\left|\left(\mathcal{P}_{3, h} z\right)^{(m)}\right|_{C^{0, \gamma}[0, h]} \leqslant C \sum_{j=0}^{m} K^{j}
$$

Using this estimate and the fact that $(Q-S)^{(m)} \in C^{0, \gamma}$ by the definitions of $Q$ and $S$, the desired estimate (4.25) follows from (4.26). This completes the proof.

By the famous Arzelà-Ascoli Theorem and Lemma 4.7, it is evident that $\mathcal{J}: V \rightarrow V$ is a compact operator, where $V$ is endowed with norm $\|\cdot\|_{C^{m}[0, h]}$. Therefore, since $V$ is a bounded, closed, convex subset of $C^{m}[0, h]$, using the Schauder Fixed-Point Theorem gives that there exists $z \in V$ such that

$$
\mathcal{J} z=z
$$




\section{Putting}

$$
y(x):=z(x)+S(x), \quad 0 \leqslant x \leqslant h,
$$

we obtain

$$
y(x)=c_{0}+\frac{1}{\Gamma(\alpha)} \int_{0}^{x}(x-t)^{\alpha-1} f(t, y(t)) \mathrm{d} t, \quad 0 \leqslant x \leqslant h .
$$

By [2, Lemma 2.1], the above $y$ is a solution of (1.1), and then, since $y^{*}$ is the unique solution of (1.1) on $\left[0, h^{*}\right]$, we have $y^{*}=y$ on $[0, h]$. Therefore, it is obvious that $y^{*}-S \in C^{m}[0, h]$ and (3.6) hold. This completes the proof of Theorem 3.1 .

\subsection{Proof of Corollary 3.1}

Let us first state the following fact. For each $1 \leqslant j \leqslant J$, by the definition of $c_{j}$, a straightforward computing yields

$$
c_{j}=\sum_{t \in \Upsilon_{j, 1} \cup \Upsilon_{j, 2}} t
$$

where

$$
\begin{aligned}
& \Upsilon_{j, 1}:=\bigcup_{\substack{1 \leqslant s<n \\
s+\alpha=\gamma_{j}}}\left\{\frac{\mathrm{B}(\alpha, 1+s) \partial_{1}^{s} f\left(0, c_{0}\right)}{\Gamma(\alpha)}\right\}, \\
& \Upsilon_{j, 2}:=\bigcup_{s=1}^{n-1} \bigcup_{\substack { k=1 \\
\begin{subarray}{c}{\beta \in \Lambda_{s} \\
\# \beta=k \\
\Gamma_{\beta} \neq \emptyset{ k = 1 \\
\begin{subarray} { c } { \beta \in \Lambda _ { s } \\
\# \beta = k \\
\Gamma _ { \beta } \neq \emptyset } }\end{subarray}}^{s}\left\{\frac{\mathrm{B}\left(\alpha, 1+s-k+\sum_{l=1}^{k} \gamma_{i_{l}}\right) \partial_{\beta} f\left(0, c_{0}\right)}{\Gamma(\alpha) \prod_{l=1}^{k} \gamma_{i_{l}}} \prod_{l=1}^{k} c_{i_{l}} \gamma_{i_{l}}:\left(i_{1}, i_{2}, \ldots, i_{k}\right) \in \Xi_{\beta, j}\right\} .
\end{aligned}
$$

Above, $\mathrm{B}(\cdot, \cdot)$ denotes the standard beta function, and

$$
\begin{gathered}
\# \beta:=\sum_{\substack{1 \leqslant i \leqslant s \\
\beta_{i}=2}} 1, \\
\Xi_{\beta, j}:=\left\{\left(i_{1}, i_{2}, \ldots, i_{\# \beta}\right): \alpha+s-\# \beta+\sum_{j=1}^{\# \beta} \gamma_{i_{j}}=\gamma_{j}\right\},
\end{gathered}
$$

for all $1 \leqslant s<n$ and $\beta \in \Lambda_{s}$.

To prove Corollary 3.1, by Theorem 3.1 it suffices to show that (3.7) is equivalent to

$$
c_{j}=0 \quad \text { for all } j \in \Theta,
$$

where

$$
\Theta:=\left\{1 \leqslant j \leqslant J: \gamma_{j} \notin \mathbb{N}\right\} .
$$

But, by (4.28), (4.29) and (4.30), an obvious induction gives

$$
c_{j}=0 \text { for all } 1 \leqslant j \leqslant J
$$

if (3.7) holds. Therefore, it remains to show that (4.31) implies (3.7).

To this end, let us assume that (4.31) holds. Note that we have (4.32). If this statement was false, then let

$$
j_{0}:=\min \left\{1 \leqslant j \leqslant J: c_{j} \neq 0\right\}
$$

Obviously, we have $j_{0}>1$ and $\gamma_{j_{0}} \in \mathbb{N}$, and in this case, $\Upsilon_{j_{0}, 1}$ is empty. Thus, by (4.28) we have

$$
c_{j_{0}}=\sum_{t \in \Upsilon_{j_{0}, 2}} t .
$$

But, by the definition of $\Upsilon_{j_{0}, 2}$ and the fact that $c_{j}=0$ for all $1 \leqslant j<j_{0}$, it is straightforward that $c_{j_{0}}=0$, which is contrary to the definition of $j_{0}$. Therefore (4.32) holds indeed. Using this result, from (4.28) and (4.30) it follows

$$
c_{j}=\sum_{t \in \Upsilon_{j, 1}} t \quad \text { for all } 1 \leqslant j \leqslant J,
$$

and then, using (4.32) again, we obtain (3.7). This completes the proof of Corollary 3.1. 


\section{Appendix A Proofs of Lemmas 4.5 and 4.6}

To prove Lemma 4.5, we need the following two lemmas.

Lemma A.1. Let $h>0, \gamma>0$ and $g \in \mathcal{C}^{1}[0, h]$. We have $w \in \mathcal{C}^{1}[0, h]$ and

$$
w^{\prime}(x)=\int_{0}^{x} t^{\gamma-1} g^{\prime}(t) \mathrm{d} t .
$$

where

$$
w(x):=\int_{0}^{x} t^{\gamma-1} g(t) \mathrm{d} t, \quad 0 \leqslant x \leqslant h .
$$

Since the proof of this lemma is straightforward, it is omitted.

Lemma A.2. Let $0<h \leqslant a$, and $l \in \mathbb{N}_{>0}$ such that $l \alpha \leqslant 1<(l+1) \alpha$. For any $g \in \mathcal{C}^{0, l \alpha}[0, h]$, we have $w \in \mathcal{C}^{0,(l+1) \alpha-1}[0, h]$ and

$$
\|w\|_{C^{0,(l+1) \alpha-1}[0, h]} \leqslant C\|g\|_{C^{0, l \alpha}[0, h]}
$$

where

$$
w(x):=\sum_{j=1}^{J} \gamma_{j} c_{j} x^{\gamma_{j}-1} g(x), \quad 0<x \leqslant h .
$$

Proof. It suffices to prove that, for any $1 \leqslant j \leqslant J$, we have $v \in \mathcal{C}[0, h]$ and

$$
\|v\|_{C^{0,(l+1) \alpha-1}[0, h]} \leqslant C\|g\|_{C^{0, l \alpha}[0, h]},
$$

where $v(x):=x^{\gamma_{j}-1} g(x), 0<x \leqslant h$. Noting the fact that $l \alpha+\gamma_{j}>1$ and $g \in \mathcal{C}^{0, l \alpha}[0, h]$, we easily obtain $v \in \mathcal{C}[0, h]$ and

$$
\|v\|_{C[0, h]} \leqslant C\|g\|_{C^{0, l \alpha}[0, h]} .
$$

It remains, therefore, to prove that

$$
|v(y)-v(x)| \leqslant C(y-x)^{(l+1) \alpha-1}\|g\|_{C^{0, l \alpha}[0, h]}
$$

for all $0<x<y \leqslant h$. Moreover, since it holds

$$
\begin{aligned}
& |v(y)-v(x)|=\left|y^{\gamma_{j}-1} g(y)-x^{\gamma_{j}-1} g(x)\right| \\
= & \left|y^{\gamma_{j}-1}(g(y)-g(x))+\left(y^{\gamma_{j}-1}-x^{\gamma_{j}-1}\right) g(x)\right| \\
\leqslant & \left(y^{\gamma_{j}-1}(y-x)^{l \alpha}+\left|y^{\gamma_{j}-1}-x^{\gamma_{j}-1}\right| x^{l \alpha}\right)\|g\|_{C^{0, l \alpha}[0, h]},
\end{aligned}
$$

by the fact $g \in \mathcal{C}^{0, l \alpha}[0, h]$, we only need to prove that

$$
y^{\gamma_{j}-1}(y-x)^{l \alpha}+\left|y^{\gamma_{j}-1}-x^{\gamma_{j}-1}\right| x^{l \alpha} \leqslant C(y-x)^{(l+1) \alpha-1}
$$

for all $0<x<y \leqslant h$.

Let us first consider the case of $\gamma_{j}<1$. A simple algebraic calculation gives

$$
\left(x^{\gamma_{j}-1}-y^{\gamma_{j}-1}\right) x^{l \alpha}=(y-x)^{l \alpha+\gamma_{j}-1}\left(A^{\gamma_{j}-1}-(1+A)^{\gamma_{j}-1}\right) A^{l \alpha},
$$

where $A:=\frac{x}{y-x}$. If $0 \leqslant A \leqslant 1$, then by the fact $l \alpha+\gamma_{j}-1>0$ we have

$$
\left(A^{\gamma_{j}-1}-(1+A)^{\gamma_{j}-1}\right) A^{l \alpha}<A^{l \alpha+\gamma_{j}-1} \leqslant 1 .
$$

If $A>1$, then using the Mean Value Theorem and the fact $l \alpha+\gamma_{j}-2<0$ gives

$$
\left(A^{\gamma_{j}-1}-(1+A)^{\gamma_{j}-1}\right) A^{l \alpha}<\left(1-\gamma_{j}\right) A^{l \alpha+\gamma_{j}-2}<\left(1-\gamma_{j}\right)<1
$$

Consequently, we obtain

$$
\left(x^{\gamma_{j}-1}-y^{\gamma_{j}-1}\right) x^{l \alpha}<(y-x)^{l \alpha+\gamma_{j}-1}
$$


which, together with the trivial estimate

$$
y^{\gamma_{j}-1}(y-x)^{l \alpha}<(y-x)^{\gamma_{j}-1}(y-x)^{l \alpha}=(y-x)^{l \alpha+\gamma_{j}-1},
$$

yields (A.2).

Then, since (A.2) is evident in the case of $\gamma_{j}=1$, let us consider the case of $1<\gamma_{j}<2$. Since $0<\gamma_{j}-1<1$, we have

$$
y^{\gamma_{j}-1}-x^{\gamma_{j}-1}<(y-x)^{\gamma_{j}-1} .
$$

By the definition of $\gamma_{j}$ it is clear that

$$
\gamma_{j}-1 \geqslant(l+1) \alpha-1
$$

Using the above two estimates, we obtain

$$
\left|y^{\gamma_{j}-1}-x^{\gamma_{j}-1}\right| x^{l \alpha} \leqslant C\left(y^{\gamma_{j}-1}-x^{\gamma_{j}-1}\right) \leqslant C(y-x)^{(l+1) \alpha-1},
$$

which, together with the estimate

$$
y^{\gamma_{j}-1}(y-x)^{l \alpha} \leqslant C(y-x)^{l \alpha} \leqslant C(y-x)^{(l+1) \alpha-1},
$$

indicates (A.2).

Finally, let us consider the case of $\gamma_{j} \geqslant 2$. Using the Mean Value Theorem gives

$$
\left|y^{\gamma_{j}-1}-x^{\gamma_{j}-1}\right| x^{l \alpha} \leqslant C(y-x)^{(l+1) \alpha-1} .
$$

and then, by the obvious estimate

$$
y^{\gamma_{j}-1}(y-x)^{l \alpha} \leqslant C(y-x)^{(l+1) \alpha-1},
$$

we obtain (A.2). This completes the proof.

Proof of Lemma 4.5 Since $g \in \mathcal{C}^{k, l \alpha}[0, h]$, by Lemma A.1 we have $w \in \mathcal{C}^{k}[0, h]$ and

$$
w^{(i)}(x)=\int_{0}^{x} \sum_{j=1}^{J} \gamma_{j} c_{j} t^{\gamma_{j}-1} g^{(i)}(t) \mathrm{d} t, \quad i=0,1,2, \ldots, k .
$$

It follows

Therefore, it remains to prove that

$$
\|w\|_{C^{k}[0, h]} \leqslant C\|g\|_{C^{k}[0, h]}
$$

$$
\left|w^{(k)}\right|_{C^{0,(l+1) \alpha}[0, h]} \leqslant C\|g\|_{C^{k, l \alpha}[0, h]}
$$

if $(l+1) \alpha \leqslant 1$; and that $w^{(k+1)} \in \mathcal{C}^{0,(l+1) \alpha-1}[0, h]$ and

$$
\left\|w^{(k+1)}\right\|_{C^{0,(l+1) \alpha-1}[0, h]} \leqslant C\|g\|_{C^{k, l \alpha}[0, h]}
$$

if $(l+1) \alpha>1$.

Let us first consider (A.4). Noting the fact that $g^{(k)} \in \mathcal{C}^{0, l \alpha}[0, h]$ and $\gamma_{j} \geqslant \alpha$ for all $1 \leqslant j \leqslant J$, by (A.3) a simple computing gives that

$$
\left|w^{(k)}(y)-w^{(k)}(x)\right| \leqslant C\left|g^{(k)}\right|_{C^{0, l \alpha}[0, h]}(y-x)^{(l+1) \alpha}
$$

for all $0 \leqslant x<y \leqslant h$, which implies (A.4).

Then let us consider (A.5). Since $g^{(k)} \in \mathcal{C}^{0, l \alpha}$, by Lemma A.2 we have $v \in \mathcal{C}^{0,(l+1) \alpha-1}[0, h]$ and

$$
\|v\|_{C^{0,(l+1) \alpha-1}[0, h]} \leqslant C\left\|g^{(k)}\right\|_{C^{0, l \alpha[0, h]}}
$$

where

$$
v(x):=\sum_{j=1}^{J} \gamma_{j} c_{j} x^{\gamma_{j}-1} g^{(k)}(x), \quad 0<x \leqslant h .
$$

Then, by (A.3) we readily obtain $w^{(k+1)} \in \mathcal{C}^{0,(l+1) \alpha-1}$ and (A.5), and thus complete the proof of this lemma.

Before proving Lemma 4.6, let us introduce the following lemma. 
Lemma A.3. Let $0<h \leqslant a$ and $\gamma>0$. For any $g \in \mathcal{C}^{k}[0, h]$ with $1 \leqslant k \leqslant m$, we have $w \in \mathcal{C}^{k-1}[0, h]$ and

$$
\|w\|_{C^{k-1}[0, h]} \leqslant C\left\|g^{(k)}\right\|_{C[0, h]},
$$

where

$$
w(x):=g(x) x^{\gamma-1}, \quad 0<x \leqslant h,
$$

and $C$ is a positive constant that only depends on $a, k$ and $\gamma$.

Proof. If $k=1$, then, by the Mean Value Theorem and the fact $g(0)=0$, this lemma is evident. Thus, below we assume that $2 \leqslant k \leqslant m$. In the rest of this proof, for ease of notation, the symbol $C$ denotes a positive constant that only depends on $a, k$ and $\gamma$, and its value may differ at each occurrence.

Let us first show that, for $0 \leqslant i<k$, we have $w_{i} \in \mathcal{C}[0, h]$ and

$$
\left\|w_{i}\right\|_{C[0, h]} \leqslant C\left\|g^{(k)}\right\|_{C[0, h]}
$$

where

$$
w_{i}(x):=w^{(i)}(x), \quad 0<x \leqslant h .
$$

To this end, let $0 \leqslant i<k$, and note that an elementary computing gives

$$
w_{i}(x)=\sum_{j=0}^{i} c_{i j} g^{(j)}(x) x^{\gamma-1-i+j}, \quad 0<x \leqslant h,
$$

where $c_{i j}$ is a constant that only depends on $\gamma, i$ and $j$, for all $0 \leqslant j \leqslant i$. Since $g \in \mathcal{C}^{k}[0, h]$, we have $g^{(j)} \in \mathcal{C}^{k-j}[0, h]$, and then, applying Taylor's formula with integral remainder yields

$$
g^{(j)}(x)=\frac{1}{(k-j-1) !} \int_{0}^{x}(x-t)^{k-j-1} g^{(k)}(t) \mathrm{d} t, \quad 0 \leqslant x \leqslant h .
$$

It follows that

$$
\left|g^{(j)}(x) x^{\gamma-1-i+j}\right| \leqslant \frac{\left\|g^{(k)}\right\|_{C[0, h]}}{(k-j) !} x^{\gamma+k-(i+1)}, \quad 0<x \leqslant h .
$$

Since $\gamma+k-(i+1) \geqslant \gamma>0$, this implies $g^{(j)}(x) x^{\gamma-i-1+j} \in \mathcal{C}[0, h]$ and

$$
\left\|g^{(j)}(\cdot)(\cdot)^{\gamma-i-1+j}\right\|_{C[0, h]} \leqslant C\left\|g^{(k)}\right\|_{C[0, h]} .
$$

Therefore, by (A.7) it follows $w_{i} \in \mathcal{C}[0, h]$ and (A.6).

Then let us proceed to prove this lemma. Let $i<k-1$. Note that by (A.7) we have

$$
w_{i}^{\prime}(x)=w_{i+1}(x), \quad 0<x \leqslant h .
$$

Since we have already proved that $w_{i}, w_{i+1} \in \mathcal{C}[0, h]$, by the Mean Value Theorem it is evident that $w_{i} \in \mathcal{C}^{1}[0, h]$ and

$$
w_{i}^{\prime}(x)=w_{i+1}(x), \quad 0 \leqslant x \leqslant h .
$$

It follows $w_{0} \in \mathcal{C}^{k-1}[0, h]$ and

$$
w_{0}^{(i)}=w_{i}, \quad 0 \leqslant i<k
$$

and hence, by (A.6) we have

$$
\left\|w_{0}\right\|_{C^{k-1}[0, h]} \leqslant C\left\|g^{(k)}\right\|_{C[0, h]} .
$$

Noting the fact $w=w_{0}$, this completes the proof.

Proof of Lemma 4.6 Below we employ the well-known principle of mathematical induction to prove this lemma. Firstly, it is clear that (4.16) holds in the case $k=0$. Secondly, assuming that (4.16) holds for $k=l$ where $0 \leqslant l<m-1$, let us prove that (4.16) holds for $k=l+1$. To this end, a straightforward computing gives

$$
\left(\mathcal{T}_{w, \beta, h} z\right)^{\prime}(x)=\mathcal{T}_{w^{\prime}, \beta, h} z(x)+\mathcal{T}_{w, \widetilde{\beta}, h} z(x)+\mathcal{T}_{\widetilde{w}, \widetilde{\beta}, h} z(x)
$$


for all $0<x \leqslant h$, where $\widetilde{\beta}:=\left(\beta_{1}, \beta_{2}, \ldots, \beta_{s}, 1\right), \widetilde{\widetilde{\beta}},:=\left(\beta_{1}, \beta_{2}, \ldots, \beta_{s}, 2\right)$, and

$$
\widetilde{w}(x):=w(x)\left(z^{\prime}(x)+\sum_{j=1}^{J} \gamma_{j} c_{j} x^{\gamma_{j}-1}\right) .
$$

Since $w \in \mathcal{C}^{k}[0, h]$, we have $w^{\prime} \in \mathcal{C}^{k-1}[0, h]$, and by Lemma A.3 we have $\widetilde{w} \in \mathcal{C}^{k-1}[0, h]$; consequently, $\mathcal{T}_{w^{\prime}, \beta, h} z$ and $\mathcal{T}_{\widetilde{w}, \widetilde{\beta}, h} z$ are well-defined, and they both belong to $\mathcal{C}[0, h]$. Therefore, by the Mean Value Theorem, and the fact $\mathcal{T}_{w, \beta, h} z \in \mathcal{C}[0, h]$, it follows that $\mathcal{T}_{w, \beta, h} z \in \mathcal{C}^{1}[0, h]$, and (A.9) holds for all $0 \leqslant x \leqslant h$. By our assumption, we have the following results: $\mathcal{T}_{w^{\prime}, \beta, h} z \in \mathcal{C}^{\min \{k-1, n-s\}}[0, h]$ and

$$
\left\|\mathcal{T}_{w^{\prime}, \beta, h} z\right\|_{C^{\min \{k-1, n-s\}}[0, h]} \leqslant C\left\|w^{\prime}\right\|_{C^{k-1}[0, h]} \sum_{j=0}^{\min \{k-1, n-s\}}\left\|z^{\prime}\right\|_{C^{m-1}[0, h]}^{j} ;
$$

$\mathcal{T}_{w, \widetilde{\beta}, h} z \in \mathcal{C}^{\min \{k-1, n-s-1\}}[0, h]$ and

$$
\left\|\mathcal{T}_{w, \widetilde{\beta}, h} z\right\|_{C^{\min \{k-1, n-s-1\}}[0, h]} \leqslant C\|w\|_{C^{k-1}[0, h]} \sum_{j=0}^{\min \{k-1, n-s-1\}}\left\|z^{\prime}\right\|_{C^{m-1}[0, h]}^{j} ;
$$

$\mathcal{T}_{\widetilde{w}, \widetilde{\beta}, h} z \in \mathcal{C}^{\min \{k-1, n-s-1\}}[0, h]$ and

$$
\left\|\mathcal{T}_{\widetilde{w}, \widetilde{\beta}, h} z\right\|_{C^{\min \{k-1, n-s-1\}}[0, h]} \leqslant C\|\widetilde{w}\|_{C^{k-1}[0, h]} \sum_{j=0}^{\min \{k-1, n-s-1\}}\left\|z^{\prime}\right\|_{C^{m-1}[0, h]}^{j} .
$$

In addition, by Lemma A.3 we easily obtain

$$
\|\widetilde{w}\|_{C^{k-1}[0, h]} \leqslant C\|w\|_{C^{k}[0, h]}\left(1+\left\|z^{\prime}\right\|_{C^{k-1}[0, h]}\right) .
$$

As a consequence, we obtain $\mathcal{T}_{w, \beta, h} z \in \mathcal{C}^{\min \{k, n-s\}}$ and

$$
\left\|\left(\mathcal{T}_{w, \beta, h} z\right)^{\prime}\right\|_{C^{\min \{k-1, n-s-1\}}[0, h]} \leqslant C\|w\|_{C^{k}[0, h]} \sum_{j=0}^{\min \{k, n-s\}}\left\|z^{\prime}\right\|_{C^{m-1}[0, h]}^{j} .
$$

Then (4.16) follows from the obvious estimate

$$
\left\|\mathcal{T}_{w, \beta, h} z\right\|_{C[0, h]} \leqslant C\|w\|_{C[0, h]} .
$$

This completes the proof of Lemma 4.6.

\section{References}

[1] K. Diethelm, Smoothness properties of solutions of Caputo-type fractional differential equations, Fract. Calc. Appl. Anal., 10 (2007), 151-160. 2

[2] K. Diethelm and N. J. Ford, Analysis of fractional differential equations, J. Math. Anal. Appl., 265 (2002), 229-249. 1, 4, 10

[3] W. Deng, Smoothness and stability of the solutions for nonlinear fractional differential equations, Nonlinear Analysis, 72 (2010), 1768-1777. 2

[4] C. Lubich, Runge-Kutta theory for Volterra and Abel integral equations of the second kind, Math. Comput., 41 (1983), 87-102. 2

[5] R. K. Miller and A. Feldstein, Smoothness of solutions of Volterra integral equations with weakly singular kernels, SIAM J. Math. Anal., 2 (1971), 242-258. 2

[6] S. G. Samko, A. A. Kilbas, and O. I. Marichev, Fractional integrals and derivatives: theory and applications, Gordon and Breach, Yverdon (1993). 6 